\title{
EDITORIAL
}

\section{AÑOS DE ENFERMERÍA UNIVERSIDAD DE CONCEPCIÓN}

\author{
70 YEARS OF NURSING UNIVERSITY OF CONCEPCIÓN \\ Dra. Viviane Jofré Aravena \\ Decana Facultad de Enfermería. Universidad de Concepción
}

Email: vjofre@udec.cl

Cada año al celebrar un año más de existencia de cualquier institución es un instante para detenerse y mirar hacia atrás, han transcurrido 70 años formando, con calidad, enfermeras/os premunidos de una sólida formación basada en los principios y valores fundacionales de la Universidad de Concepción.

La formación de enfermeras/os en Concepción surgió como respuesta a la necesidad de formar profesionales con una visión integral de la atención de enfermería dentro del proceso salud-enfermedad. La antigua Escuela de Enfermería perteneció, en sus inicios, a la Beneficencia Pública que era dirigida por el médico penquista y posterior Rector de esta Universidad Ignacio González Ginouvés, quien propuso en 1947 a Concepción como sede para la formación de enfermeras fuera de Santiago, dado el prestigio que la Universidad ya ostentaba en esa fecha. Durante varios años se mantuvo con el carácter de Programa de Salud bajo la tutela de la Universidad de Chile, egresando en enero de 1951 una cohorte de 18 enfermeras, después de tres años y medio de estudio.

En 1955 pasó a depender académicamente de nuestra Universidad y administrativamente del Servicio Nacional de Salud dependiente del Ministerio respectivo.

En 1959 se realiza una reestructuración, con un nuevo programa de formación que se extiende a cuatro años, el que posteriormente se ampliaría a cuatro años y medio y en el año 1996 a la duración actual de 5 años, obteniendo la Licenciatura en Enfermería el año anterior.

A partir del 15 de julio de 1970 es incorporada como Escuela de Enfermería, en plenitud a nuestra Universidad y realiza sus funciones en base a departamentos en las siguientes áreas: Fundamentos de Enfermería, Enfermería Materna y Enfermería del Adulto. 
Sin lugar a dudas uno de los hitos más importantes es haber creado los programas de especialidad para enfermeras en la década de los 80, al igual que el Programa de Magíster en Enfermería y posteriormente la creación del primer Doctorado en Enfermería en el país en el año 2004 (1).

Paralelo a lo anterior, la Revista Ciencia y Enfermería se ha constituido en un referente en investigación para nuestro país y Latinoamérica, desde hace más de 20 años (2).

La autonomía académica y administrativa adquirida, al constituirnos en abril de 2015 en Facultad de Enfermería, ha sido un fuerte impulso para todas nuestras iniciativas; es así como en la actualidad se imparte un plan de estudios innovado, teniendo presente las tendencias internacionales, los contextos sociales, políticos y jurídicos, los nuevos estándares de acreditación, las exigencias del mercado y el desarrollo estratégico institucional de nuestra Facultad y de nuestra Casa de Estudios.

Innegablemente es momento, además, para dirigir la mirada al futuro planteándonos nuevos desafíos para responder a las demandas de los diferentes escenarios, culturales, sociales, económicos, tecnológicos y políticos, que sin duda han ido determinando cambios en nuestro rol profesional, otorgándole en sus inicios un eminente énfasis en el rol curativo de nuestro accionar para adoptar posteriormente un giro preventivo e integral, exigiéndose así una formación con más herramientas teóricas para una práctica más especializada, haciendo que las responsabilidades se ampliaran y complejizaran.

Esta unidad académica de enfermería ha recorrido un camino largo y fructífero, con el esfuerzo de todos quienes la integraron e integran actualmente; aunque no sólo el esfuerzo, sino que además la disciplina, el compromiso y la excelencia de cada uno de los integrantes de nuestra organización nos permite mirar al futuro con optimismo. Hemos sido capaces de escribir esta historia y de vivir momentos que nos llenan de alegría y emociones que pintan los estados del alma.

Seguramente irán apareciendo nuevos retos porque, tal como lo plantea Nájera, los profesionales enfermeras/os debemos ser conscientes de que el análisis continuo y sistemático del quehacer profesional permitirá detectar aquellas lagunas del conocimiento sustantivas y metodológicas que no pueden ser resueltas con el estudio de la información existente; es indispensable que toda interrogante que surja del trabajo día a día deberá constituirse en reflexiones y actividades prioritarias de investigación (3), para lograr contribuir a mejorar las condiciones de salud de la población, aportar elementos innovadores en la atención sanitaria e incidir en las políticas científicas e institucionales para facilitar el empoderamiento de la profesión con su entorno social. 


\section{REFERENCIAS}

1. Jofré-Aravena V, Paravic-Klijn T. Postgrado en Enfermería en Chile. Index Enferm. 2018; 16(56): 50-54.

2. Mendoza Parra S. Ciencia y Enfermería 20 años después. Cienc. enferm. 2015; $\mathrm{XXI}(3): 7-9$.

3. Nájera RM. La Asociación Latinoamericana de Escuelas y Facultades de Enfermería y su aportación a la docencia, la investigación y la práctica en enfermería. Revista Iberoamericana de Educación e Investigación en Enfermería. 2011; 1(1): 12-16. 